\title{
Urine-albumin and creatinine ratio among apparently healthy individual in South Eastern, Nigeria
}

Amos Dangana ${ }^{1,}{ }^{*}$, Ifeoma Miracle Okoronkwo ${ }^{1}$, Solomon Oloche Onoja ${ }^{1}$, Innocent Nwabueze Okonkwo ${ }^{3}$ Nonye Bibiana Egenti ${ }^{2}$, Shalom Chidiogo Azolike ${ }^{1}$, Ovye Engom Alaba ${ }^{1}$ and Mustapha Bakare ${ }^{1}$

${ }^{1}$ Medical laboratory Services, University of Abuja Teaching Hospital Gwagwalada, Nigeria.

2 Department of Community Medicine University of Abuja Teaching Hospital Gwagwalada, Nigeria.

${ }^{3}$ Department of Medical Laboratory Science, University of Nigeria, Nsukka.

GSC Biological and Pharmaceutical Sciences, 2021, 16(01), 105-114

Publication history: Received on 27 May 2021; revised on 04 July 2021; accepted on 08 July 2021

Article DOI: https://doi.org/10.30574/gscbps.2021.16.1.0185

\begin{abstract}
Background: Albuminuria and Albumin-Creatinine Ratio (ACR) has been identified as early indices of cardiovascular diseases (CVD), Hypertension and are at great risk of hypertensive nephropathy, but this not so for the apparently healthy individuals.
\end{abstract}

Objectives: This study was therefore carried out to evaluate and quantitate albuminuria and urine ACR among apparently healthy individual and to compare values with the hypertensive attending University of Nigeria Teaching Hospital, Ituku-Ozalla Enugu State.

Materials and method: The study was carried out on eighty-nine (89) volunteers (50 tests and 39 controls) age 21-77 years between August and September 2019. A structured questionnaire was used to get vital information, anthropometric measurements were taken while blood and urine samples were collected and analyzed for serum creatinine (Scr), urine creatinine (UCr) and urine albumin using Jaffe modified kinetic method and Enzyme Linked Immunosorbent Assay (ELISA) method respectively. Body mass index (BMI) in $\mathrm{Kg} / \mathrm{m}^{2}$, urine ACR in $\mathrm{mg} / \mathrm{mmol}$ and estimated glomerular filtration rate (eGFR) using Chronic Disease Epidemiology Equation were calculated. Data were analyzed using student's T-test and one-way Analysis of variance (ANOVA).

Results: Generally, results showed that males have higher $(\mathrm{p}<0.05)$ height, Scr, eGFR, and UCr but lower ( $<<0.05)$ BMI) when compared with the females, but no significant difference $(\mathrm{P}>0.05)$ between systolic blood pressure (SBP), diastolic BP (DBP), pulse, weight, urine albumin and ACR. Also, the study revealed that the test subjects have higher SBP, DBP, weight, BMI, SCr, urine albumin, and ACR but lower $(\mathrm{p}<0.05)$ eGFR and UCr when compared with the control subjects, but no significant difference $(\mathrm{P}>0.05)$ between pulse and height. Furthermore, intra gender analysis of the results showed that males tests have higher SBP, DBP, weight, BMI and urine ACR but lower $(\mathrm{p}<0.05)$ eGFR and UCr when compared with male controls, but no significant difference $(\mathrm{P}>0.05)$ was observed for pulse, height, Scr and urine albumin. Finally, the results showed that female tests have higher $(\mathrm{P}<0.05) \mathrm{SBP}$, pulse, Scr, urine albumin and ACR but lower $(\mathrm{p}<0.05)$ eGFR when compared with the female controls

Conclusion: Findings and results from this study suggest that the hypertensives stand a higher risk of kidney damage, consequently cardiovascular disease (CVD).

Keywords: Urine, Albumin, Creatinine, Ratio, Apparently Healthy

\footnotetext{
* Corresponding author: Amos Dangana

Medical laboratory Services University of Abuja Teaching Hospital Gwagwalada, Nigeria.
} 


\section{Introduction}

Albuminuria (microalbuminuria) also knowns urinary albumin-creatinine ratio (UACR) is defined as excretion of 30$300 \mathrm{mg}$ of albumin per 24 hours (or $20-200 \mathrm{mcg} / \mathrm{min}$ or $30-300 \mathrm{mcg} / \mathrm{mg}$ creatinine) on 2 of 3 urine collections or $\geq 3.5$ $\mathrm{mg} / \mathrm{mmol}$ (female) or $\geq 2.5 \mathrm{mg} / \mathrm{mmol}$ (male). Alternatively, albuminuria (microalbuminuria) may be defined as urinary albumin-creatinine ratio (UACR) on a random urine sample of more than $30 \mathrm{mg}$ (but less than $300 \mathrm{mg}$ ) of albumin per gram of creatinine (Levin et al., 2012). The presence of albuminuria even at low levels still remains a vital marker of chronic kidney disease and may also predicts end-stage kidney disease. Importantly, albuminuria independently predicts cardiovascular diseases with lethal outcome; this may be attributed endothelial dysfunction (Olsen et al., 2016). Traditionally, albuminuria, rather than overall proteinuria, has been utilized in studies of progression in studies with CKD in adults, although the association of normal or mildly increased levels of albuminuria on worsening renal function has been explored in the adult CKD population (Gansevoort et al., 2011).

Measurement of urinary albumin excretion to assess this risk requires the collection of a timed urine collection is cumbersome and prone to error because of inadequate urine collections (Olsen et al., 2016). More so, the reference standard of 24-hour urine collection is impractical in many settings due to prolong sampling (Matsushita et al., 2010). To overcome these problems, it has been proposed to evaluate UAER by measuring the urinary albumin-to-creatinine ratio (UACR) in a spot urine sample. This test was adopted because of its simplicity and is recommended by various guidelines (Dana et al., 2017). Urine ACR and spot urine albumin concentration reliably estimate 24-hour albumin excretion, although the correlation is stronger for ACR because it accounts for differences in urine concentration due to hydration status, additionally, postural changes interferes with albumin excretion (Stevens et al., 2013). However, because the timing of collection tends not to correlate with explanatory variables of interest such as socioeconomic status or age, this does not introduce confounding (Huan, et al., 2016). Consequently, random samples are routinely used for research purposes also and most evidence relating albuminuria to kidney and cardiovascular risk derives from random urine ACR (Nicholas et al., 2019).

Among adults, given the clear relationship between albuminuria and future cardiovascular events or chronic kidney disease, there has been a shift in focus from pathological levels to the risk associated with urine ACR levels in the lownormal range (Arie et al., 2011). The Chronic Kidney Disease Prognosis Consortium has shown through collaborative meta-analysis that a urine ACR of $1.1 \mathrm{mg} / \mathrm{mmol}$ is associated with a hazard ratio of 1.20 for all-cause mortality (95\% CI 1.15 to 1.26 ) compared with a urine ACR of $0.6 \mathrm{mg} / \mathrm{mmol}$ (Matsushita et al., 2010). Such relationships, where risk extends below traditional thresholds into the normal range, are common among clinical biomarkers and make it difficult to define the threshold at which values should be considered abnormal (Abdul-Rahman and Ibrahiem, 2014).

Microalbuminuria occurs in $11 \%$ to $40 \%$ of hypertensive patients. Interestingly, the prevalence increases with age and the duration of hypertension (Steven et al., 2006). In a cross-sectional study of more than 10,000 nondiabetic patients with hypertension, patients with microalbuminuria had a significantly higher prevalence of cardiovascular disease symptoms and outcomes compared with normo albuminuric subjects (Ghonemy et al., 2016). Further, microalbuminuria was found to be associated with increased risk for cardiovascular disease in high-risk men with treated hypertension, and this risk is associated with small increases in albuminuria that may not even register as microalbuminuria. This increased risk seems to start at relatively low levels of albuminuria (Larkins et al., 2017).

Microalbuminuria has been reported in $6.1 \%$ of men and $9.7 \%$ of women in the general population in the United States without diabetes, hypertension, cardiovascular disease, or elevated creatinine. European studies report a $5 \%$ to $7 \%$ prevalence of microalbuminuria in the general population (Steven et al., 2006).

Owing to the fact that sustained hypertension is one of the leading course of chronic kidney disease (CKD), it has become of most importance to monitor renal functionality amongst the hypertensive, therefore this study aim at examining renal functions among apparently healthy individuals and to compare values with the hypertensive subjects. This will help in drawing out a benchmark values among the renal functioning parameters which will aid in the treatment course of the hypertensive and to abate the progression of CKD into end state CKD. Present study will also help in the addition of knowledge to the scientific community at large. 


\section{Material and methods}

\subsection{Study Area}

The study was carried out in the University of Nigeria Teaching Hospital (U.N.T.H.), Enugu South-East Nigeria. The hospital is located 22 kilometres from Enugu the capital along the Enugu Port Harcourt expressway and covers an area of 200 acres and serves mainly the rural and urban areas of Enugu, as well as referrals from the neighbouring states of Anambra, Abia, Ebonyi, Imo, Delta, Benue and Kogi.

\subsection{Study Population}

The study was carried out on a total of 89 volunteers ( 50 test group and 39 control group) from the University of Nigeria Teaching Hospital Ituku-Ozalla age 21-77 years.

\subsection{Study Design}

It was a case-control and cross-sectional study.

\subsection{Data Collection}

A structured questionnaire was used to get medical history as well as other relevant data from the participant, including informed consent. Absolute confidentiality was maintained.

\subsection{Participants Sampling Technique}

Samples was collected by random sampling from hypertensive subjects attending the University of Nigeria Teaching Hospital Ituku-Ozalla, Enugu while the control sample was collected from apparently healthy individuals that are not hypertensive.

\subsection{Sample collection, preparation and storage}

About 15 millilitres (ml) of early morning spot urine was collected into a clean, chemically free wide neck transparent container and the urine was stored at $4^{0} \mathrm{c}$ in a plain tube for micro-albumin and urine creatinine assay. Blood was collected from the participants using a sterile $2 \mathrm{ml}$ needle and syringe which was transferred into a sterile labelled plain vial. The blood in the plain vials was allowed to clot followed by clot retraction and the sample centrifuged at 5000 revolutions per minutes for 5 minutes and the serum was dispensed into a serum container and stored at $4^{\circ} \mathrm{C}$ for serum creatinine assay.

\section{Laboratory analytical procedure}

\subsection{Serum Creatinine}

Biochemical analyses were performed on the serum specimen using Humalyzer 2000 chemistry semi auto-analyzer (Human GmBHDiagnostica Worldwide, Wiesbaden, Germany). The modified Jaffe kinetic method was used as modified by Cromatest kit. Results interpreted as described by the kit's manufacture instructions.

\subsection{Urine Micro-Albumin}

A biochemical analysis was performed on the urine using Mindray 96A Enzyme-Linked Immunosorbent Assay (ELISA) Microplate Reader was used (Mindray Bio-Medical Electronics Company, Shenzhen, China). The concentration of microalbumin in the samples is then determined by comparing the optical density of the samples to the standard curve.

\subsection{Estimation of glomerular filtration rate}

Serum creatinine values were used to estimate glomerular filtration rate (GFR) using the Chronic Kidney Disease Epidemiology Equation (CKD-EPI) formula.

\subsection{Anthropometric measurements}

The following anthropometric data were measured: height, weight, and blood pressure. Standing height was measured to the nearest centimetre $(\mathrm{cm})$ using a stadiometer with footwear removed. Weight was measured to the nearest kilogram (kg) with a manual Seca 761 scale (Vogel and Halke, Hamburg, Germany) after participants have removed 
outer garments, cell phone, and footwear. Blood pressure (BP) and pulse rate were measured with an automated sphygmomanometer (OMRON HEM705CP, Omron Matsusaka Co, Matsusaka city, Mie-Ken, Japan) using appropriate cuff size after participants have sat undisturbed for at least 5 minutes. Three consecutive readings were taken one minute apart, and the mean of the three readings was used for the analysis. Body mass index (BMI) was calculated using the metric body mass index formulae weight $(\mathrm{kg}) /[\text { height }(\mathrm{m})]^{2}$.

\subsection{Statistical Analysis}

Data obtained from this study were analyzed using the statistical package for social sciences (SPSS) version 22 of Windows IBM Inc. Chicago, IL, USA. Data were presented as means and standard deviations. Student's t-test was used to calculate differences between means, Analysis of Variance (ANOVA) was used to estimate differences between group means. All hypotheses +tests were performed using two-tailed and p value $<0.05$ considered statistically significant.

\section{Results}

Generally, results showed that males have higher $(\mathrm{p}<0.05)$ height, serum creatinine, eGFR, and urine creatinine but lower $(\mathrm{p}<0.05)$ BMI $\left(1.71 \pm 0.08 \mathrm{~m}, 102.11 \pm 13.38 \mu \mathrm{mol} / \mathrm{L}, 87.16 \pm 15.74 \mathrm{ml} / \mathrm{min} / 1.73 \mathrm{~m}^{2}, 13.25 \pm 2.30 \mathrm{mmol} / \mathrm{L}\right.$, and $26.39 \pm 4.44 \mathrm{Kg} / \mathrm{m}^{2}$ respectively) when compared with the females $(1.63 \pm 0.08 \mathrm{~m}, 88.00 \pm 14.06 \mu \mathrm{mol} / \mathrm{L}, 78.66 \pm 19.54$ $\mathrm{ml} / \mathrm{min} / 1.73 \mathrm{~m}^{2}, 10.94 \pm 1.03 \mathrm{mmol} / \mathrm{L}$, and $30.59 \pm 5.45 \mathrm{Kg} / \mathrm{m}^{2}$ respectively), but no significant difference (P $>0.05$ ) between systolic blood pressure (SBP), diastolic BP (DBP), pulse, weight, urine albumin and ACR. The study revealed that the test subjects have higher SBP, DBP, weight, BMI, serum creatinine, urine albumin, and ACR but lower (p<0.05) eGFR and urinary creatinine $\left(139.96 \pm 22.14 \mathrm{mmHg}, 82.68 \pm 13.69 \mathrm{mmHg}, 83.00 \pm 14.82 \mathrm{Kg}, 29.80 \pm 4.70 \mathrm{Kg} / \mathrm{m}^{2}\right.$, $99.02 \pm 14.42 \mu \mathrm{mol} / \mathrm{L}, 13.83 \pm 5.44 \mathrm{mg} / \mathrm{L}, 1.25 \pm 0.48 \mathrm{mg} / \mathrm{mmol}, 73.58 \pm 14.93 \mathrm{ml} / \mathrm{min} / 1.73 \mathrm{~m}^{2}$, and $11.06 \pm 1.37 \mathrm{mmol} / \mathrm{L}$ respectively) when compared with the control subjects $(113.46 \pm 9.93 \mathrm{mmHg}, 72.36 \pm 7.25 \mathrm{mmHg}, 74.44 \pm 13.18 \mathrm{Kg}$, $26.75 \pm 5.74 \mathrm{Kg} / \mathrm{m}^{2}, 90.15 \pm 15.03 \mu \mathrm{mol} / \mathrm{L}, 11.09 \pm 5.02 \mathrm{mg} / \mathrm{L}, 13.45 \pm 2.17 \mathrm{mmol} / \mathrm{L}, 94.97 \pm 14.49 \mathrm{ml} / \mathrm{min} / 1.73 \mathrm{~m}^{2}$, and $13.45 \pm 2.17 \mathrm{mmol} / \mathrm{L}$ respectively), but no significant difference $(\mathrm{P}>0.05)$ between pulse and height. Furthermore, intra gender analysis of the results showed that males tests have higher SBP, DBP, weight, BMI and urine ACR but lower $(\mathrm{p}<0.05)$ eGFR and urinary creatinine $\left(148.18 \pm 17.88 \mathrm{mmHg}, 86.77 \pm 12.94 \mathrm{mmHg}, 84.91 \pm 14.68 \mathrm{Kg}, 28.43 \pm 4.49 \mathrm{Kg} / \mathrm{m}^{2}\right.$, $1.27 \pm 0.42 \mathrm{mg} / \mathrm{mmol}, 80.73 \pm 16.42 \mathrm{ml} / \mathrm{min} / 1.73 \mathrm{~m}^{2}$, and $11.42 \pm 1.62 \mathrm{mmol} / \mathrm{L}$ respectively) when compared with male controls $\left(112.96 \pm 8.35 \mathrm{mmHg}, 70.22 \pm 7.46 \mathrm{mmHg}, 70.39 \pm 9.59 \mathrm{Kg}, 24.45 \pm 3.47 \mathrm{Kg} / \mathrm{m}^{2}, 0.83 \pm 0.30 \mathrm{mg} / \mathrm{mmol}, 93.30 \pm 12.54\right.$ $\mathrm{ml} / \mathrm{min} / 1.73 \mathrm{~m}^{2}, 14.99 \pm 1.24 \mathrm{mmol} / \mathrm{L}$ respectively), but no significant difference $(\mathrm{P}>0.05)$ was observed for pulse, height, Scr and urine albumin. Finally, the results showed that female tests have higher $(\mathrm{P}<0.05) \mathrm{SBP}$, pulse, Scr, urine albumin and ACR but lower ( $<<0.05)$ eGFR $(133.50 \pm 23.30 \mathrm{mmHg}, 76.18 \pm 7.35 \mathrm{bpm}, 95.18 \pm 12.03 \mu \mathrm{mol} / \mathrm{L}, 13.40 \pm 6.04$ $\mathrm{mg} / \mathrm{L}, 1.24 \pm 0.53 \mathrm{mg} / \mathrm{mmol}$, and $67.96 \pm 10.97 \mathrm{ml} / \mathrm{min} / 1.73 \mathrm{~m}^{2}$ respectively) when compared with the female controls $(114.19 \pm 12.11 \mathrm{mmHg}, 69.38 \pm 7.99 \mathrm{bpm}, 75.44 \pm 6.47 \mu \mathrm{mol} / \mathrm{L}, 9.53 \pm 4.96 \mathrm{mg} / \mathrm{L}, 0.86 \pm 0.47 \mathrm{mg} / \mathrm{mmol}$, and $97.38 \pm 17.06$ $\mathrm{ml} / \mathrm{min} / 1.73 \mathrm{~m}^{2}$ respectively).

\section{Discussion}

Hypertension (HT) is the second leading cause of kidney failure. In hypertensive patients with chronic kidney disease (CKD), blood pressure (BP) control is the most important intervention to minimize progression (Salvador-González et al., 2017). Healthy kidneys produce physiologically appropriate urine, it retain essential blood components while excreting waste materials. Excess albumin in the urine, or albuminuria, has been regarded as a principal marker for renal disease (Zhang et al., 2011), physiologically, more albumin appears in the urine when the kidneys are no longer functioning properly (Metra et al., 2012). From our results, table 1.

shows statistically significant increase in the urine creatinine (U Creat.), eGFR, serum creatinine (S. Creat.) and height among male participants with P-values of $0.000,0.026,0.000$ and 0.000 respectively, while statistically non-significant increase was seen in systolic BP, diastolic BP, pulse rate, urine albumin and urine ACR with p-value $\geq 0.05$. Furthermore, statistically significant decrease was seen in BMI with p-value of 0.000 among male subjects. Table 1 also shows statistically significant decrease in height, serum creatinine, eGFR and urine creatinine among female subjects with pvalues of $0.000,0.000,0.026$ and 0.000 among female patients.

Our findings were in keeping with a particular study where the mean age was 15.5 years, and older adolescents appear to have a lower prevalence of albuminuria (Boyd et al., 2013). More so both the Australian Health Survey (AHS) and National Health and Nutrition Examination Survey (NHANES) studies, females had a higher ACR than males because of differences in urine albumin, rather than differences in urine creatinine concentration. The most likely explanation for this is that normal physiological values vary by sex, rather than sex-related differences in cardiovascular or kidney 
health at this age (Bobby and Sitar 2001). The prevalence of albuminuria and mean values for urine ACR, urine albumin and urine creatinine concentrations were similar in children and adults (Dana et al., 2017).

Table 1 Mean \pm SD of Age, Systolic BP, Diastolic BP, Pulse, Weight, Height, BMI, Serum Creatinine, eGFR, Urine Albumin, Urine Creatinine and Urine ACR of the Males and Females

\begin{tabular}{|l|c|c|c|c|}
\hline & All (n=89) & Male (n=45) & Female (n=44) & \multirow{2}{*}{ p-value } \\
\cline { 1 - 4 } & Mean \pm SD & Mean \pm SD & Mean \pm SD & \\
\hline Age (year) & $50.81 \pm 14.73$ & $48.76 \pm 14.98$ & $52.91 \pm 14.33$ & 0.185 \\
\hline Systolic BP (mmHg) & $128.35 \pm 22.12$ & $130.18 \pm 22.46$ & $126.48 \pm 21.91$ & 0.434 \\
\hline Diastolic BP (mmHg) & $78.16 \pm 12.39$ & $78.31 \pm 13.34$ & $78.00 \pm 11.50$ & 0.907 \\
\hline pulse (BPM) & $73.75 \pm 9.03$ & $73.80 \pm 9.88$ & $73.70 \pm 8.20$ & 0.961 \\
\hline Weight (Kg) & $79.25 \pm 14.68$ & $77.49 \pm 14.24$ & $81.05 \pm 15.07$ & 0.256 \\
\hline Height (M) & $1.67 \pm 0.09$ & $1.71 \pm 0.08$ & $1.63 \pm 0.08$ & 0.000 \\
\hline BMI((Kg/m $\left.{ }^{2}\right)$ & $28.47 \pm 5.37$ & $26.39 \pm 4.44$ & $30.59 \pm 5.45$ & 0.000 \\
\hline Serum creatinine( $\mu \mathrm{mol} / \mathrm{L})$ & $95.13 \pm 15.38$ & $102.11 \pm 13.38$ & $88.00 \pm 14.06$ & 0.000 \\
\hline eGFR(ml/min/1.73m $\left.{ }^{2}\right)$ & $82.96 \pm 18.13$ & $87.16 \pm 15.74$ & $78.66 \pm 19.54$ & 0.026 \\
\hline Urine albumin(mg/L) & $12.63 \pm 5.41$ & $13.26 \pm 4.84$ & $11.99 \pm 5.92$ & 0.271 \\
\hline Urine Creatinine(mmol/L) & $12.11 \pm 2.12$ & $13.25 \pm 2.30$ & $10.94 \pm 1.03$ & 0.000 \\
\hline Urine ACR (mg/mmol) & $1.07 \pm 0.49$ & $1.05 \pm 0.44$ & $1.01 \pm 0.53$ & 0.595 \\
\hline
\end{tabular}

Table 2 Mean \pm SD of Age, Systolic BP, Diastolic BP, Pulse, Weight, Height, BMI, Serum Creatinine, eGFR, Urine Albumin, Urine Creatinine and Urine ACR of the Test and Control Subjects

\begin{tabular}{|l|c|c|c|c|}
\hline & All (n=89) & Test (n=50) & Control (n=39) & p-value \\
\hline & Mean \pm SD & Mean \pm SD & Mean \pm SD & \\
\hline Age (year) & $50.81 \pm 14.73$ & $57.40 \pm 9.73$ & $42.36 \pm 15.81$ & 0.000 \\
\hline Systolic BP (mmHg) & $128.35 \pm 22.14$ & $139.96 \pm 22.14$ & $113.46 \pm 9.93$ & 0.000 \\
\hline Diastolic BP (mmHg) & $78.16 \pm 12.39$ & $82.68 \pm 13.69$ & $72.36 \pm 7.25$ & 0.000 \\
\hline pulse (BPM) & $73.75 \pm 9.03$ & $74.66 \pm 9.16$ & $72.59 \pm 8.85$ & 0.286 \\
\hline Weight (Kg) & $79.25 \pm 14.68$ & $83.00 \pm 14.82$ & $74.44 \pm 13.18$ & 0.006 \\
\hline Height (M) & $1.67 \pm 0.09$ & $1.67 \pm 0.10$ & $1.67 \pm 0.08$ & 0.683 \\
\hline BMI((Kg/m $\left.{ }^{2}\right)$ & $28.47 \pm 5.37$ & $29.80 \pm 4.70$ & $26.75 \pm 5.74$ & 0.007 \\
\hline Serum creatinine( $\left.\mu \mathrm{mol}^{\mathrm{L}} \mathrm{L}\right)$ & $95.13 \pm 15.38$ & $99.02 \pm 14.42$ & $90.15 \pm 15.03$ & 0.006 \\
\hline eGFR(ml/min/1.73m $\left.{ }^{2}\right)$ & $82.96 \pm 18.13$ & $73.58 \pm 14.93$ & $94.97 \pm 14.49$ & 0.000 \\
\hline Urine albumin(mg/L) & $12.63 \pm 5.41$ & $13.83 \pm 5.44$ & $11.09 \pm 5.02$ & 0.017 \\
\hline Urine Creatinine(mmol/L) & $12.11 \pm 2.12$ & $11.06 \pm 1.37$ & $13.45 \pm 2.17$ & 0.000 \\
\hline Urine ACR (mg/mmol) & $1.07 \pm 0.49$ & $1.25 \pm 0.48$ & $0.84 \pm 0.40$ & 0.000 \\
\hline
\end{tabular}

This was unexpected because, as a marker of early disease and risk for future clinical cardiovascular and kidney events, we would expect an increase in the prevalence of albuminuria between childhood and mid-adulthood, as is observed 
between young and older adults (ABS, 2013). Our findings shows statistically non-significant increases in U. ACR among male participants when compared with the female counterpart, this is in disparity with earlier findings where observed sex discrepancy in ACR was noticed among females which explains their higher prevalence of albuminuria despite a slightly lower prevalence of risk factors such as hypertension and obesity (Nicholas et al., 2017).

From our results in table 2, there was statistically significant increase among control group (apparently healthy individuals) in eGRF and urine creatinine when compared with the test group (hypertensive subjects) with p-values of 0.000 and 0.000 respectively. while statistically significant decrease was reported for age, systolic blood pressure (SBP), diastolic blood pressure (DBP), weight, body mass index (BMI), serum creatinine (S. creat.), eGFR, urine albomine (U Alb), urine albumin and creatinine ratio (U ACR) with p-value $<0.05$.

Table 3 Mean \pm SD of Age, Systolic BP, Diastolic BP, Pulse, Weight, Height, BMI, Serum Creatinine, eGFR, Urine Albumin, Urine Creatinine and Urine ACR of the Male Tests and Male Controls

\begin{tabular}{|l|c|c|c|c|}
\hline & All (n=45) & Test (n=22) & Control (n=23) & p-value \\
\hline & Mean \pm SD & Mean \pm SD & Mean \pm SD & \\
\hline Age (year) & $48.76 \pm 14.98$ & $57.27 \pm 9.41$ & $40.61 \pm 14.92$ & 0.000 \\
\hline Systolic BP (mmHg) & $130.18 \pm 22.46$ & $148.18 \pm 17.88$ & $112.96 \pm 8.35$ & 0.000 \\
\hline Diastolic BP (mmHg) & $78.31 \pm 13.34$ & $86.77 \pm 12.94$ & $70.22 \pm 7.46$ & 0.000 \\
\hline pulse (BPM) & $73.80 \pm 9.88$ & $72.73 \pm 10.92$ & $74.83 \pm 8.90$ & 0.482 \\
\hline Weight (Kg) & $77.49 \pm 14.24$ & $84.91 \pm 14.68$ & $70.39 \pm 9.59$ & 0.000 \\
\hline Height (M) & $1.71 \pm 0.08$ & $1.73 \pm 0.09$ & $1.70 \pm 0.07$ & 0.231 \\
\hline BMI((Kg/m $\left.{ }^{2}\right)$ & $26.39 \pm 4.44$ & $28.43 \pm 4.49$ & $24.45 \pm 3.47$ & 0.002 \\
\hline Serum creatinine( $\mu \mathrm{mol} / \mathrm{L})$ & $102.11 \pm 13.38$ & $103.91 \pm 15.96$ & $100.39 \pm 10.42$ & 0.384 \\
\hline eGFR(ml/min/1.73m $\left.{ }^{2}\right)$ & $87.16 \pm 15.74$ & $80.73 \pm 16.42$ & $93.30 \pm 12.54$ & 0.006 \\
\hline Urine albumin(mg/L) & $13.26 \pm 4.84$ & $14.39 \pm 4.64$ & $12.18 \pm 4.87$ & 0.127 \\
\hline Urine Creatinine(mmol/L) & $13.25 \pm 2.30$ & $11.42 \pm 1.62$ & $14.99 \pm 1.24$ & 0.000 \\
\hline Urine ACR (mg/mmol) & $1.05 \pm 0.44$ & $1.27 \pm 0.42$ & $0.83 \pm 0.30$ & 0.000 \\
\hline
\end{tabular}

Our findings were similar to previous study by Tarek et al. who reported that prevalence of cardiovascular disease events in elderly patients with eGFR between 39 and $59 \mathrm{~mL} / \mathrm{min}$ is more than in population with eGFR > $60 \mathrm{~mL} / \mathrm{min}$ and also that there was more deterioration of eGFR with increase in age (Salvador-González et al., 2017). Our findings were also in conformity with the Simone et al. who noted reduced eGFR among Patients with Hypertensive Nephropathy and Chronic Kidney Disease (Simone et al.2018). More so, it was noted from previous report that decline in eGFR was association with higher SBP, DBP and MAP. Furthermore, there were statistically significant interactions between the BP components and antihypertensive treatment, so that the associations between BP and GFR decline occurred primarily in patients treated with antihypertensive agents. The reason may be that GFR is more sensitive to BP changes in treated persons because longstanding hypertension or antihypertensive drugs interfere with the autoregulation of glomerular blood flow. This makes GFR more sensitive to changes in BP, which manifested as a steeper GFR decline with intensified BP treatment in our population (Eriksen et al.2017).

Similar trend from table 2 were observed in both table 3 and 4, on the contrary, they were marked disparity in an Australian study, were population-based $13.5 \%$ of adults and $15.1 \%$ of children had albuminuria, and the prevalence varied by sex, with albuminuria more common in females among both children and, especially, adults. In children, the sex difference was mostly driven by an increased urine albumin concentration, whereas in adults the urine creatinine concentration was relatively more important. There was weak intergenerational concordance for urine albumin and ACR, but creatinine concordance was larger (Larkins, et al. 2017). Similar findings were also documented in a study where significant correlation was observed between gender and CKD. The prevalence of CKD is generally higher in women (Ravera et al., 2011), but when adjusted for other risk factors, male gender is associated with the presence of CKD (Salvador et al., 2015). In another study, the association between BP and the risk of CKD in women was weaker 
than in men among the hypertensive untreated group. This may be caused by gender-related differences, such as estrogen production, which has been suggested to prevent atherosclerosis (Arnal et al., 2004). These findings may raise the hypothesis that the deleterious effect of elevated BP on the kidneys may be weaker in women. However, no study has clarified the gender difference and the effect of intensive treatment on kidney protection (Satoh et al., 2020)

Table 4 Mean \pm SD of Age, Systolic BP, Diastolic BP, Pulse, Weight, Height, BMI, Serum Creatinine, eGFR, Urine Albumin, Urine Creatinine and Urine ACR of the Female Tests and Female Controls

\begin{tabular}{|l|c|c|c|c|}
\hline & All (n=44) & Test (n=28) & Control (n=16) & p-value \\
\hline & Mean \pm SD & Mean \pm SD & Mean \pm SD & \\
\hline Age (year) & $50.91 \pm 14.32$ & $57.50 \pm 10.15$ & $44.88 \pm 17.18$ & 0.004 \\
\hline Systolic BP (mmHg) & $126.48 \pm 21.91$ & $133.50 \pm 23.30$ & $114.19 \pm 12.11$ & 0.004 \\
\hline Diastolic BP (mmHg) & $78.00 \pm 11.50$ & $79.46 \pm 13.62$ & $75.44 \pm 5.86$ & 0.289 \\
\hline pulse (BPM) & $73.70 \pm 8.20$ & $76.18 \pm 7.35$ & $69.38 \pm 7.99$ & 0.007 \\
\hline Weight (Kg) & $81.05 \pm 15.07$ & $81.50 \pm 15.03$ & $80.25 \pm 15.61$ & 0.795 \\
\hline Height (M) & $1.63 \pm 0.08$ & $1.62 \pm 0.07$ & $1.64 \pm 0.09$ & 0.350 \\
\hline BMI((Kg/m $\left.{ }^{2}\right)$ & $30.59 \pm 5.45$ & $30.89 \pm 4.65$ & $30.07 \pm 6.77$ & 0.638 \\
\hline Serum creatinine( $\mu \mathrm{mol} / \mathrm{L})$ & $88.00 \pm 14.60$ & $95.18 \pm 12.03$ & $75.44 \pm 6.47$ & 0.000 \\
\hline eGFR(ml/min/1.73m $\left.{ }^{2}\right)$ & $78.66 \pm 19.54$ & $67.96 \pm 10.97$ & $97.38 \pm 17.06$ & 0.000 \\
\hline Urine albumin(mg/L) & $11.99 \pm 5.92$ & $13.40 \pm 6.04$ & $9.53 \pm 4.96$ & 0.036 \\
\hline Urine Creatinine(mmol/L) & $10.94 \pm 1.03$ & $10.78 \pm 1.08$ & $11.23 \pm 0.88$ & 0.173 \\
\hline Urine ACR (mg/mmol) & $1.10 \pm 0.53$ & $1.24 \pm 0.53$ & $0.86 \pm 0.47$ & 0.019 \\
\hline
\end{tabular}

Table 5 Mean \pm SD of Age, Systolic BP, Diastolic BP, Pulse, Weight, Height, BMI, Serum Creatinine, eGFR, Urine Albumin, Urine Creatinine and Urine ACR of the Test Males and Test Females

\begin{tabular}{|l|c|c|c|c|}
\hline & All (n=50) & Male (n=22) & Female (n=28) & p-value \\
\hline & Mean \pm SD & Mean \pm SD & Mean \pm SD & \\
\hline Age (year) & $57.40 \pm 9.73$ & $57.27 \pm 9.41$ & $57.50 \pm 10.15$ & 0.963 \\
\hline Systolic BP (mmHg) & $139.96 \pm 22.14$ & $148.18 \pm 17.88$ & $133.50 \pm 23.30$ & 0.018 \\
\hline Diastolic BP (mmHg) & $82.68 \pm 13.69$ & $86.77 \pm 12.95$ & $79.46 \pm 13.61$ & 0.060 \\
\hline pulse (BPM) & $74.66 \pm 9.16$ & $72.73 \pm 10.92$ & $76.18 \pm 7.35$ & 0.189 \\
\hline Weight (Kg) & $83.00 \pm 14.82$ & $84.91 \pm 14.68$ & $81.50 \pm 15.03$ & 0.425 \\
\hline Height (M) & $1.67 \pm 0.10$ & $1.73 \pm 0.09$ & $1.62 \pm 0.07$ & 0.000 \\
\hline BMI((Kg/m $\left.{ }^{2}\right)$ & $29.80 \pm 4.70$ & $28.43 \pm 4.49$ & $30.89 \pm 4.65$ & 0.066 \\
\hline Serum creatinine( $\mu \mathrm{mol} / \mathrm{L})$ & $99.02 \pm 14.42$ & $103.91 \pm 15.96$ & $95.18 \pm 12.03$ & 0.032 \\
\hline eGFR(ml/min/1.73m $\left.{ }^{2}\right)$ & $73.58 \pm 14.93$ & $80.73 \pm 16.42$ & $67.96 \pm 10.97$ & 0.002 \\
\hline Urine albumin(mg/L) & $13.83 \pm 5.44$ & $14.39 \pm 4.64$ & $13.40 \pm 6.04$ & 0.528 \\
\hline Urine Creatinine(mmol/L) & $11.06 \pm 1.37$ & $11.42 \pm 1.62$ & $10.78 \pm 1.08$ & 0.104 \\
\hline Urine ACR (mg/mmol) & $1.25 \pm 0.48$ & $1.27 \pm 0.42$ & $1.24 \pm 0.53$ & 0.840 \\
\hline
\end{tabular}


In addition, reduced UCr has been found to be associated with major cardiovascular events and mortality in the general population (Oterdoom et al., 2009), similar findings also noted long-term data on the rate of decline of UCr in patients with CKD stages 3-5 and its association with clinical outcomes (Micco et al., 2013). Statistically significant increase in urine creatinine noted in our findings could be attributed to the normal physiologic optimal renal performance of the apparently healthy individuals.

Variations noticed among male and female control in table 6 could be attributed to physiologic effect of sex hormones. Sex hormones affect the structure of renal tubules and various aspects of renal functions in experimental animals and humans. Due to contradictory data on the presence, abundance, and intracellular localization of sex hormone receptors in renal tubules, it is presently impossible to correlate gender dependent expression of these receptors with renal functions and expression of various transporters (Sabolić et al., 2007).

In the table 2, it can be observed that there was a statically significant in the age, systolic BP, diastolic BP, weight, BMI, serum creatinine, eGFR, urine albumin, urine creatinine and urine ACR with (P value $<0.05)$ when the test subjects were compared with the control subjects.

In the table 3, it can be observed that there was a statically significant in the age, systolic BP, diastolic BP, weight, BMI, eGFR, urine creatinine and urine ACR with (P value $<0.05)$ when the male tests were compared with the male controls.

In the table 4 it can be observed that there was a statically significant in the age, systolic BP, pulse, serum creatinine, eGFR, urine albumin and urine ACR with (P value < 0.05) when the female tests were compared with the female controls.

In the table 5 it can be observed that there was a statically significant in the systolic blood pressure, height, serum creatinine and eGFR with (P value $<0.05$ ) when test males and test females were compared.

In the table 6 it can be observed that there was a statically significant in Diastolic blood pressure, weight, height, BMI, serum creatinine and urine creatinine with (P value $<0.05)$ when male controls were compared with female controls.

Table 6 Mean \pm SD of Age, Systolic BP, Diastolic BP, Pulse, Weight, Height, BMI, Serum Creatinine, eGFR, Urine Albumin, Urine Creatinine and Urine ACR of the Control Males and Control Females

\begin{tabular}{|l|c|c|c|c|}
\hline & All (n=39) & Male (n=23) & Female (n=16) & p-value \\
\hline & Mean \pm SD & Mean \pm SD & Mean \pm SD & \\
\hline Age (year) & $42.36 \pm 15.81$ & $40.61 \pm 14.92$ & $44.88 \pm 17.18$ & 0.414 \\
\hline Systolic BP (mmHg) & $113.46 \pm 9.93$ & $112.96 \pm 8.35$ & $114.19 \pm 12.11$ & 0.709 \\
\hline Diastolic BP (mmHg) & $72.36 \pm 7.25$ & $70.22 \pm 7.46$ & $75.44 \pm 5.86$ & 0.025 \\
\hline pulse (BPM) & $72.59 \pm 8.85$ & $74.83 \pm 8.90$ & $69.38 \pm 7.99$ & 0.057 \\
\hline Weight (Kg) & $74.44 \pm 13.18$ & $70.39 \pm 9.59$ & $80.25 \pm 15.61$ & 0.019 \\
\hline Height (M) & $1.67 \pm 0.08$ & $1.70 \pm 0.07$ & $1.64 \pm 0.09$ & 0.027 \\
\hline BMI((Kg/m $\left.{ }^{2}\right)$ & $26.75 \pm 5.74$ & $24.45 \pm 3.47$ & $30.07 \pm 6.77$ & 0.002 \\
\hline Serum creatinine( $\left.\mu \mathrm{mol}^{\mathrm{L}} \mathrm{L}\right)$ & $90.15 \pm 15.30$ & $100.39 \pm 10.42$ & $75.44 \pm 6.47$ & 0.000 \\
\hline eGFR(ml/min/1.73m $\left.{ }^{2}\right)$ & $94.97 \pm 14.49$ & $93.30 \pm 12.54$ & $97.38 \pm 17.06$ & 0.395 \\
\hline Urine albumin(mg/L) & $11.09 \pm 5.02$ & $12.18 \pm 4.87$ & $9.53 \pm 4.96$ & 0.106 \\
\hline Urine Creatinine(mmol/L) & $13.45 \pm 2.17$ & $14.99 \pm 1.24$ & $11.23 \pm 0.88$ & 0.000 \\
\hline Urine ACR (mg/mmol) & $0.84 \pm 0.40$ & $0.83 \pm 0.36$ & $0.86 \pm 0.47$ & 0.861 \\
\hline
\end{tabular}

\section{Conclusion}

Findings and results from this study suggest that the hypertensives stand a higher risk of kidney damage, consequently cardiovascular disease (CVD). 


\section{Compliance with ethical standards}

\section{Disclosure of conflict of interest}

All authors declare that they have no conflict of interest.

\section{Statement of informed consent}

Informed consent was obtained from all individual participants included in the study.

\section{References}

[1] Nicholas GL, Siah K, John BC, Anneke CG, David PB, Katherine L, et al. Albuminuria: population epidemiology and concordance in Australian children aged 11-12 years and their parents. BMJ Open. 2019; 9: 75-84.

[2] Matsushita K, van der Velde M, Astor BC, et al. Association of estimated glomerular filtration rate and albuminuria with all-cause and cardiovascular mortality in general population cohorts: a collaborative meta-analysis. Lancet. 2010; 375: 2073-81. 7. Rademacher ER, Sinaiko AR. Albuminuria in children. Curr Opin Nephrol Hypertens. 2009; 18: 246-51.

[3] Larkins N, Teixeira-Pinto A, Craig J. The population-based prevalence of albuminuria in children. Pediatr Nephrol. 2017; 32: 2303-9.

[4] Olsen MH, Angell SY, Asma S, et al. A call to action and a lifecourse strategy to address the global burden of raised blood pressure on current and future generations: the Lancet Commission on hypertension. Lancet. 2016; 388: 2665-712.

[5] Boyd A, Golding J, Macleod J, et al. Cohort Profile: the 'Children of the 90s'--the index offspring of the Avon Longitudinal Study of Parents and Children. Int J Epidemiol. 2013; 42: 111-27.

[6] Australian Bureau of Statistics (ABS). Australian Health Survey: Biomedical Results for Chronic Diseases, 201112. Canberra: ABS. 2013.

[7] Clayton P, Hurst K. ANZDATA Registry Report. Adelaide: Australia and New Zealand Dialysis and Transplant Registry. 2016.

[8] Dana YF, Michael FS, Katherine MD, Tom DB, Robert M, Jeffrey MS, et al. Albuminuria, Proteinuria, and Renal Disease Progression in Children with CKD. Clin J Am Soc Nephrol. 2017; 12: 912-920.

[9] Gansevoort RT, Matsushita K, van der Velde M, Astor BC, Woodward M, Levey AS, et al. Chronic Kidney Disease Prognosis Consortium: Lower estimated GFR and higher albuminuria are associated with adverse kidney outcomes. A collaborative meta-analysis of general and high-risk population cohorts. Kidney Int. 2011; 80: 93104.

[10] Levin A, Stevens PE: Summary of KDIGO. 2012 CKD Guideline: Behind the scenes, need for guidance, and a framework for moving forward. Kidney Int. 2014; 85: 49-61.

[11] Huan L, Yuezhong L, Chao W, HaiTao T. The urine albumin-to-creatinine ratio is a reliable indicator for evaluating complications of chronic kidney disease and progression in IgA nephropathy in China. Clinics. 2016; 71(5): 243250

[12] Stevens PE, Levin A. Kidney Disease: Improving Global Outcomes CKD Guideline Development Work Group M. Evaluation and management of CKD: synopsis of the kidney disease: improving global outcomes 2012 clinical practice guideline. Ann Intern Med. 2013; 158(11): 825-30,

[13] Arie E, Ruth R, Tiki M, Rachel SL, Janos W, Iskar D. et al. The Urine ACR: Assessment of Its Performance in the Renal Transplant Recipient Population. Clin J Am Soc Nephrol. 2011; 6.

[14] Steven PC, Tanya MO, Wayne DC. Albuminuria: It's Importance in Disease Detection. Journal of labmedicine: 2007.

[15] Abdul-Rahman IS. Urine Albumin/creatinine ratio: A reliable marker of renal injury in sickle cell nephropathy. Saudi J Med Med Sci. 2014; 2: 17-23.

[16] Ghonemy TA, Ebrahim MS, Soliman SA, Allam HA. Reduced glomerular filtration rate as a predictor of coronary artery disease events in elderly patients. Alexandria Journ. Of Med. 2016. 
[17] Metra1 M, Cotter G, Gheorghiade M, Dei Cas L, Voors AA. The role of the kidney in heart failure. European Heart Journal. 2012; 33: 2135-2143.

[18] Zhang R, Zheng L, Sun Z, Zhang X, Li J, Hu D, et al. Decreased glomerular filtration rate is associated with mortality and cardiovascular events in patients with hypertension: a prospective study. PLoS One. 2011; 6(11): e27359.

[19] Salvador-González B, Mestre-Ferrer J, Soler-Vila M, Pascual-Benito L, Alonso-Bes E, Cunillera-Puértolas O, et al. Enfermedad renal crónica en individuos hipertensos $\geq 60$ anos atendidos en Atención Primaria. Nefrologia. 2017; 37: 406-414.

[20] Salvador GB, Rodríguez PM, Ruipérez GL, Ferré GA, Cunillera PO, Rodríguez LM. Enfermedad renal crónica en Atención Primaria: prevalencia y factores de riesgo asociados. Aten Primaria. 2015; 47: 236-45.

[21] Ravera M, Noberasco G, Weiss U, Re M, Gallina AM, Filippi A, et al. CKD awareness and blood pressure control in the primary care hypertensive population. Am J Kidney Dis. 2011; 57: 71-7.

[22] Eriksen BO, Stefansson VT, Jenssen TG, Mathisen UD, Schei J, Solbu MD, et al. Blood pressure and age-related GFR decline in the general population. BMC Nephrology. 2017; 18: 77.

[23] Oterdoom LH, Gansevoort RT, Schouten JP, de Jong PE, Gans ROB, Bakker SJL: Urinary creatinine excretion, an indirect measure of muscle mass, is an independent predictor of cardiovascular disease and mortality in the general population. Atherosclerosis. 2009; 207: 534-540.

[24] Micco LD, Quinn RR, Ronksley PE, Bellizzi V, Lewin AM Cianciaruso B, et al. Urine Creatinine Excretion and Clinical Outcomes in CKD. 2013: Clin J Am Soc Nephrol. 2013; 8: 1877-1883.

[25] Bobby B, Sitar DS. The effect of lactate on sex differences in rat renal tubular energy-dependent transport of the organic cation amantadine. Pharmacology. 2001; 62: 188-192.

[26] Sabolić I, Asif AR, Budach WE, Wanke C, Bahn A, Burckhardt G. Gender differences in kidney function. Pflugers Arch - Eur J Physiol. 2007; 455: 397-429.

[27] Satoh M, Hirose T, Nakayama S, Murakami T, Takabatake T, Asayama K, et al. Blood Pressure and Chronic Kidney Disease Stratified by Gender and the Use of Antihypertensive Drugs. J Am Heart Assoc. 2020; 9: e015592.

[28] Arnal JF, Gourdy P, Elhage R, Garmy-Susini B, Delmas E, Brouchet L, Castano C, Barreira Y, Couloumiers JC, Prats $\mathrm{H}$, et al. Estrogens and atherosclerosis. Eur J Endocrinol. 2004; 150: 113-117. 